\title{
Fluorescence studies of the interaction of pyrenylmethyl tributylphosphonium bromide with double-strand polynucleotides
}

\author{
M. Elisabete C. D. Real Oliveira, * Adelina L. F. Baptista, Paulo J. G. Coutinho, \\ Elisabete M. S. Castanheira and Graham Hungerford \\ Departamento de Física, Universidade do Minho, Campus de Gualtar, 4710-057 Braga, \\ Portugal.E-mail: beta@fisica.uminho.pt
}

Received 13th May 2003, Accepted 5th November 2003

First published as an Advance Article on the web 1st December 2003

\begin{abstract}
The interaction between pyren-1-ylmethyl tri-n-butylphosphonium bromide (PMTP), a water-soluble cationic pyrene derivative and the double-strand polynucleotides Poly[dA-dT], Poly[dA]·Poly[dT], Poly[dG-dC] and Poly[dG]• Poly[dC] was studied using UV-Vis absorption and fluorescence spectroscopy. The PMTP probe interacts with polynucleotides through both weak and intercalative binding, evidenced through changes in the absorption spectrum (hypochromicity and red shift). The two binding types were distinguished using time-resolved fluorescence, as the intercalative environment differs from that of the surface. Thymine and cytosine are more efficient quenchers of PMTP situated on the surface because of the higher proton accessibility to this region. In contrast, adenine does not quench PMTP fluorescence, whereas guanine residues are always very efficient quenching sites. Therefore, through the use of spectroscopic techniques, it was possible to obtain information concerning the partition of PMTP in each form of binding. In the heteropolymers, Poly[dA-dT] and Poly[dG-dC], PMTP exhibits a significant preference for intercalation in AT sequences, while with GC, the intercalation is lower. In the homopolymers, Poly[dA]·Poly[dT] and Poly $[\mathrm{dG}] \cdot$ Poly $[\mathrm{dC}]$, the main mechanism of interaction is weak binding, but some base preferences are elucidated. Additionally, Poly[dA] Poly[dT] can be distinguished from Poly[dA-dT] through a direct energy-transfer process between several bound PMTP molecules.
\end{abstract}

\section{Introduction}

There are numerous planar aromatic compounds that intercalate with nucleic acids and their highly conjugated ring systems, and the mechanism of interaction of polynuclear aromatic hydrocarbon derivatives with DNA has been of interest for some time and dates back to $1946 .{ }^{1-11}$ Many cationic substituted polycyclic aromatic hydrocarbons, such as those derived from anthracene and pyrene, generally bind to DNA non-specifically by intercalation between base pairs, ${ }^{9}$ but there is some evidence indicating a preference for alternating dA-dT sequences over alternating $\mathrm{dG}-\mathrm{dC}$ sequences. ${ }^{7,9,12-15}$ This base pair selectivity is possibly influenced by variations in local structure attributed to electrostatic interactions with the DNA bases. It has been reported that the fluorescence of some aromatic hydrocarbons is strongly quenched by an interaction between the dyes and the nucleobase, and steady progress has been made over many years towards the interpretation of fluorescence data from intercalating molecules that contain the pyrene moiety. ${ }^{5-8,10,15,16}$

The strong absorption and fluorescence characteristics of PMTP (pyrenylmethyl tri-n-butylphosphonium bromide), a water-soluble cationic pyrene derivative, provide a sensitive spectroscopic probe with which to study interactions with both single- and double-strand polynucleotides. This compound has proved to be very useful when compared with other pyrene derivatives because of its overall structure and positive charge. PMTP is water soluble but retains some of the characteristics of pyrene, like sensitivity to the surrounding environment and the ability to form excimers. Previous work performed with this probe, showed that it can induce the growth of mixed nanoaggregates with anionic surfactants. ${ }^{17,18}$ PMTP has also been employed with the polyelectrolyte heparin and polynucleotides; this work indicated that both intercalation and electrostatic interactions may be involved in the binding of this probe to nucleic acids. ${ }^{19}$
In a previous paper, ${ }^{20}$ interactions between PMTP, singlestrand polynucleotides and single-strand DNA were described. Here, three distinguishable binding modes were uncovered, namely, electrostatic binding to the phosphate group, which occurs mostly at low ionic strength, intercalative binding between bases and surface (groove) binding. In order to better understand the effect of the double helix in the binding of PMTP to DNA, we choose some representative synthetic double-strand polynucleotides, Poly[dG]·Poly[dC], Poly[dA]· Poly[dT], Poly[dG-dC] and Poly[dA-dT]. As the interaction between charged species and DNA is of importance, ${ }^{14,21,22}$ it was hoped that this study on the partitioning of PMTP between binding sites, with a particular focus on Poly[dA-dT], may complement other pyrene studies and contribute to a deeper understanding of the interactions of such compounds with DNA.

\section{Materials and methods}

Reagents

Poly[dG]·Poly[dC], Poly[dA]·Poly[dT], Poly[dG-dC] and Poly[dA-dT] were obtained from Boehringer (Lewes, UK). Polynucleotide solutions were prepared in $1 \times 10^{-3} \mathrm{M}$ sodium phosphate buffer ( $\mathrm{pH}$ 7.2) with $1 \times 10^{-4} \mathrm{M}$ EDTA and either with or without $0.2 \mathrm{M}$ sodium chloride. The concentration of nucleic acids in these solutions was determined from their decadic molar extinction coefficients at $260 \mathrm{~nm} .{ }^{23}$ Pyrenylmethyl tri-n-butylphosphonium bromide (structure shown below) was synthesised as described elsewhere ${ }^{24}$ and has a decadic molar extinction coefficient of $32000 \mathrm{M}^{-1} \mathrm{~cm}^{-1}$ at 344 $\mathrm{nm}$ in aqueous solution.

A stock solution of PMTP $\left(0.2 \mathrm{mg} \mathrm{mL}^{-1}\right)$ was prepared in $1 \times 10^{-3} \mathrm{M}$ phosphate buffer at $\mathrm{pH} 7.2$ and stored in darkness at $6{ }^{\circ} \mathrm{C}$. Aliquots of this solution (from 100 to $500 \mu \mathrm{L}$ ) were made up to $2.5 \mathrm{~mL}$ with phosphate buffer and with the appropriate concentration of $\mathrm{NaCl}$. 


\section{PMTP}

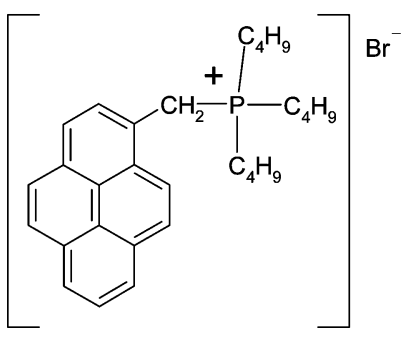

Steady-state measurements

Absorption spectra were recorded using a Shimadzu UV3101PC spectrophotometer and steady-state fluorescence spectra were obtained with a Spex Fluorolog 212 spectrofluorometer. The temperature of samples was maintained at $22{ }^{\circ} \mathrm{C}$ and all measurements were carried out using airequilibrated solutions.

\section{Time-resolved fluorescence measurements}

Time-resolved fluorescence measurements were performed using a single-photon counting spectrometer. This was equipped with a coaxial flashlamp excitation source with a full width at half-maximum of around $1.7 \mathrm{~ns}$. The excitation wavelength was selected using a monochromator $(16 \mathrm{~nm}$ band pass). Detection at $90^{\circ}$ to the excitation, selected via filters, was acheived using a Hamamatsu R2949 side-window photomultiplier. The time calibration was 231 ps per channel and the instrumental response function was recorded sequentially after the decay using a scattering solution. Data were analysed by a sum of exponentials of the form

$$
I(t)=\sum_{i=1}^{n} \alpha_{i} \exp \left(-t / \tau_{i}\right)
$$

The goodness of fit was judged in terms of the $\chi^{2}$ value weighted residuals and the autocorrelation function. Al measurements were performed at $22{ }^{\circ} \mathrm{C}$ without degassing of sample solutions.

\section{Binding data}

McGhee-Von Hippel analysis ${ }^{25}$ was performed on Scatchardtype data with the commercial plotting package Delta Graph (RockWare Inc., Golden, CO, USA) using a LevenbergMarquardt fitting routine. The procedures for obtaining the Scatchard data have been described elsewere. ${ }^{20}$

\section{Molecular modelling}

Molecular modelling was performed using Hyperchem 7 software (Hypercube Inc., Gainesville, FL, USA) applying an AMBER2 force to a model consisting of thirty base pairs of the system being studied, plus one molecule of PMTP situated near to either the minor or major groove, or intercalated. Energies were taken from structures that had been geometrically optimised and annealing was also performed to check for the presence of local energy minima.

\section{Results and discussion}

\section{PMTP in aqueous solution}

In order to clarify the behaviour of PMTP in double-strand polynucleotide solutions, the spectroscopic features of this pyrene derivative in aqueous solution were investigated (Table 1).
PMTP in aqueous solution (with or without $\mathrm{NaCl}$ ) at low concentration $\left(2 \times 10^{-6} \mathrm{M}\right)$ exhibits the typical fluorescence spectrum for pyrene in aqueous media. The ratio of the fluorescence intensity of the first $(0 \longleftarrow 0)$ and third $(2 \longleftarrow 0)$ vibronic bands, $\mathrm{I}_{1} / \mathrm{I}_{3}$, is 1.86 , close to the value reported for pyrene in water by Dong and Winnik. ${ }^{26}$ The fluorescence decay is monoexponential, with a lifetime of $128.5 \mathrm{~ns}$, which is typical for the intrinsic lifetime of a pyrene derivative in the presence of oxygen. ${ }^{27}$ At a higher concentration $\left(1 \times 10^{-5} \mathrm{M}\right)$, the PMTP fluorescence spectrum exhibits a very slight amount of excimer emission. In the absence of $\mathrm{NaCl}$, the excimer decay is biexponential, with a risetime of $11.5 \mathrm{~ns}$ and a decay time of $53 \mathrm{~ns}\left(\chi^{2}=1.17\right)$. However, the ratio of pre-exponential factors is far from -1 , which gives an indication of the occurrence of ground-state pyrene dimer formation. In solution, the excited pre-associated dimer of pyrene can rapidly revert to the excimer and the direct emission of the excited dimer may not be observed. ${ }^{28}$ The monomer decay is triexponential and decay times of 11.5 and $53 \mathrm{~ns}$, along with $128 \mathrm{~ns}$ for the major component, are obtained $\left(\chi^{2}=1.1\right)$. The latter shows that there are a few isolated PMTP molecules in solution that do not participate in the monomer-excimer equilibrium. This may relate to the repulsive effect of the positive charge, opposing some of the molecular encounters.

In the presence of $0.1 \mathrm{M} \mathrm{NaCl}$, the risetime encountered in the biexponential excimer decay disappears and a short decay component of $2.17 \mathrm{~ns}$ is seen (the other component found is $63 \mathrm{~ns}$, along with a goodness of fit of $\chi^{2}=1.06$ ). The new short decay time can be attributed to the decay of an excited dimer pre-associated in the ground state. The presence of $\mathrm{NaCl}$ has the effect of screening the repulsion between the positively charged phosphonium groups of PMTP, hence, favouring the aggregation of the pyrenyl derivative in the ground state which, in turn, reduces the interconversion of dimers to excimers.

\section{PMTP in polynucleotide solutions}

The absorption and fluorescence spectra of PMTP change significantly in solutions with double-strand polynucleotides. The excited-state properties of pyrene and pyrene derivatives are very sensitive to the environment and exhibit pronounced spectral changes, such as fluorescence quenching, spectral shifts and excimer or exciplex formation. These provide a sensitive spectroscopic means by which to study the interaction between the chromophore and DNA base pairs. ${ }^{29}$

PMTP-Poly[dA-dT] interactions. Fluorescence spectra of $\operatorname{PMTP}\left(1.5 \times 10^{-5} \mathrm{M}\right)$ at different concentrations of Poly[dA-dT] are presented in Fig. 1 (the inset shows the absorption spectra under the same conditions). The relation between the PMTP and polynucleotide concentrations can be expressed in terms of the $[\mathrm{P}] /[\mathrm{D}]$ ratio, where $\mathrm{P}$ stands for phosphate (number of bases) and D for dye (in this case, PMTP).

With polynucleotide addition, a decrease in absorption ( $\sim 5 \%$ hypochromicity) is observed, along with a $9 \mathrm{~nm}$ red shift of the main absorbance peak (from 344 to $353 \mathrm{~nm}$ ), as well as the formation of an isobestic point at $350 \mathrm{~nm}$. These changes in the absorption spectrum are similar to those observed for pyrene and other pyrene derivatives in the presence of DNA. ${ }^{12,13,15,30}$ This suggests that the pyrenyl rings are closely associated with the bases. At high ionic strength $(0.2 \mathrm{M} \mathrm{NaCl})$ the absorption spectra also display hypochromism and a $9 \mathrm{~nm}$ red shift. We chose to use the isobestic point in the absorption spectra as the excitation wavelength for fluorescence spectra. The emission in the absence of $\mathrm{NaCl}$ (Fig. 1) exhibits a reduction in PMTP fluorescence $(\sim 70 \%)$ with increasing Poly[dA-dT] concentration. There is also a dramatic change in the spectral shape, a red shift of about $5 \mathrm{~nm}$ is observed and the vibrational structure is completely different. At $[\mathrm{P}] /[\mathrm{D}]$ values near 3 , a new emitting species is clearly seen to form as PMTP binds to the 
Table 1 Fluorescence parameters for PMTP at different concentrations in $1 \mathrm{mM}$ air-saturated phosphate buffer (pH 7.2) in the presence and absence of $\mathrm{NaCl} ; \lambda_{\mathrm{exc}}=344 \mathrm{~nm}, \lambda_{\mathrm{em}}=400 \mathrm{~nm}$

\begin{tabular}{|c|c|c|c|c|c|}
\hline$[\mathrm{PMTP}] / \mathrm{M}$ & {$[\mathrm{NaCl}] / \mathrm{M}$} & $\lambda_{\mathrm{em}}$ & $\mathrm{I}_{1} / \mathrm{I}_{3}$ & Fluorescence lifetime/ns & Reduced $\chi^{2}$ \\
\hline \multirow[t]{4}{*}{$2 \times 10^{-6}$} & 0 & 400 & \multirow{3}{*}{1.86} & 128.5 & 1.1 \\
\hline & 0 & & & & \\
\hline & 0.1 & 400 & & 128.5 & 1.05 \\
\hline & 0.1 & & \multirow{6}{*}{$\begin{array}{l}1.86 \\
1.82\end{array}$} & & \\
\hline \multirow[t]{5}{*}{$1.5 \times 10^{-5}$} & 0 & 400 & & $11.5(5.3 \%), 53(0.5 \%), 128(94.2 \%)$ & 1.1 \\
\hline & 0 & & & & \\
\hline & 0 & 480 & & $11.5(-0.12), 53(0.53)$ & 1.17 \\
\hline & 0.1 & 400 & & $1.98(27.4 \%), 62.9(42.1 \%), 128(30.5 \%)$ & 1.09 \\
\hline & 0.1 & 480 & & $2.17(37 \%), 63(63 \%)$ & 1.06 \\
\hline
\end{tabular}

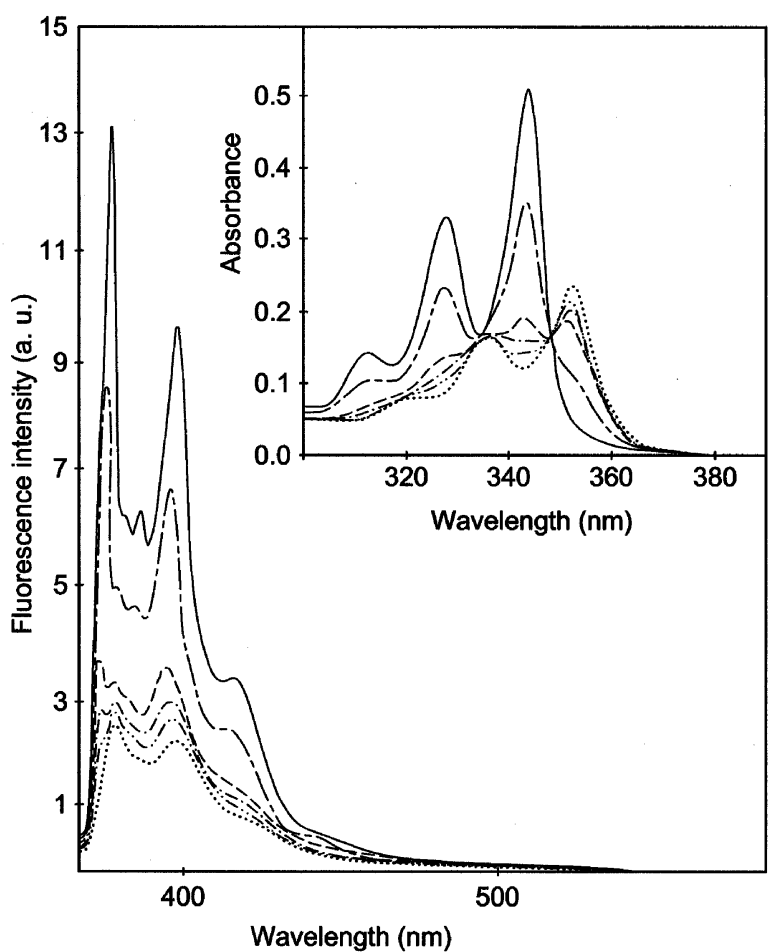

Fig. 1 Fluorescence spectra $\left(\lambda_{\text {exc }}=350 \mathrm{~nm}\right)$ for PMTP $\left(1.5 \times 10^{-5} \mathrm{M}\right)$ in Poly[dA-dT] solutions containing $1 \times 10^{-3} \mathrm{M}$ phosphate buffer $(\mathrm{pH} 7.2)$ at different $[\mathrm{P}] /[\mathrm{D}]$ ratios: $(-) 0 ;(--)$ ) $1.0 ;(--) 3.0 ;(--)) 3.5$ $(-\cdot-) 5.0 ;(\cdots)$ 7.0. The inset shows absorption spectra at the same $[\mathrm{P}] /[\mathrm{D}]$ ratios.

nucleotides. At $[\mathrm{P}] /[\mathrm{D}]=7$, the lower wavelength vibronic band of the PMTP emission disappears, indicating a high degree of binding. These features in the absorption and emission spectra are rather different to those observed when significant groundstate pyrene aggregation takes place, as already reported for aqueous solutions of pyrene-labelled hydrophobically modified polymers ${ }^{31}$ and for SDS premicellar aggregates. ${ }^{17}$

Fig. 2 illustrates the changes in the PMTP emission spectrum for different excitation wavelengths; here, in a Poly[dA-dT] solution at $[\mathrm{P}] /[\mathrm{D}]=5$. An enlarged view of the longer wavelength emission is shown in the inset. When $\lambda_{\mathrm{exc}}=344 \mathrm{~nm}$, the emission is not very different to that of free PMTP in aqueous solution, but the $\mathrm{I}_{1} / \mathrm{I}_{3}$ intensity ratio in the monomer region is lower (1.57). With $\lambda_{\text {exc }}=354$ and $260 \mathrm{~nm}$, the spectra exhibit the features shown in Fig. 1 for higher $[\mathrm{P}] /[\mathrm{D}]$ values. At $260 \mathrm{~nm}$, the optical density of polynucleotide bases is $\sim 40$ times greater than that of PMTP. The fluorescence from the bases is negligible as the quantum yield is very low $\left(\sim 10^{-4}\right){ }^{32}$ As the maximum wavelength of nucleotide emission occurs at 310-320 $\mathrm{nm},{ }^{23,32}$ an energy-transfer sensitisation process of PMTP by the polynucleotide bases is possible. The Förster radius should be small due to the very low fluorescence yield of the nucleotides. Thus, the close coincidence of the PMTP fluorescence spectra in the region below $420 \mathrm{~nm}$ when using excitation wavelengths of 354 or $260 \mathrm{~nm}$ provides good

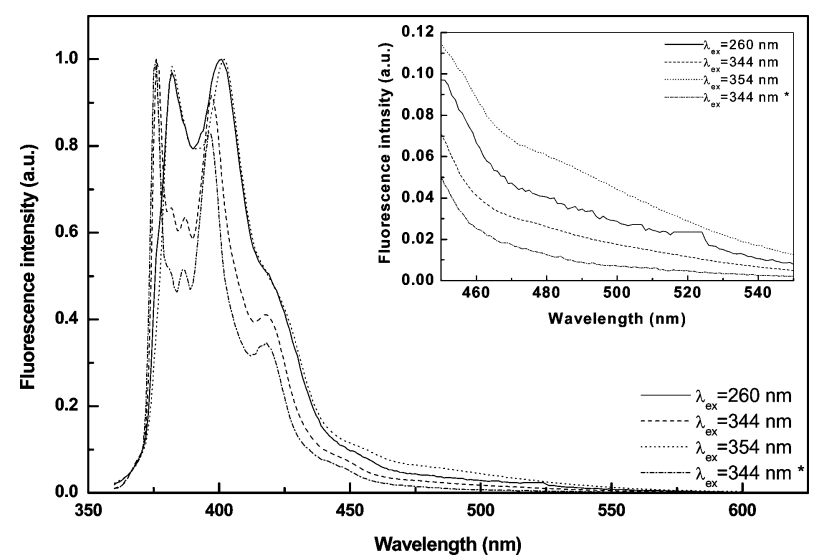

Fig. 2 Emission spectra of PMTP $\left(1.5 \times 10^{-5} \mathrm{M}\right)$ in Poly[dA-dT] solution $([\mathrm{P}] /[\mathrm{D}]=5)$ with $1 \times 10^{-3} \mathrm{M}$ phosphate buffer $(\mathrm{pH} 7.2)$ at different excitation wavelengths. The emission spectrum of free PMTP (*) at $\lambda_{\mathrm{ex}}=344 \mathrm{~nm}$ is shown for comparison. The inset shows an expansion of the $450-550 \mathrm{~nm}$ region.

evidence for the formation of an intercalation complex between PMTP and the Poly[dA-dT] bases. When $\lambda_{\text {exc }}=344 \mathrm{~nm}$, where free PMTP is preferentially excited, the spectrum reflects a coexistence between free and bound species. At this $[\mathrm{P}] /[\mathrm{D}]$ (see Fig. 2), it can be seen that there are some unbound PMTP molecules. Also, a small broad band is visible around $480 \mathrm{~nm}$, which is typical of an excimer-type species. The intensity of this band (relative to that for the monomer) was found to vary with excitation wavelength and was most pronounced at $\lambda_{\mathrm{exc}}=354$ $\mathrm{nm}$ (see inset in Fig. 2). This provides evidence for ground-state pyrene aggregation. In fact, it is in the longer wavelength region that the relative dimer to monomer absorption ratio should be higher, since the pyrene $\mathrm{S}_{1} \leftarrow \mathrm{S}_{0}$ band has a very low absorption coefficient.

The excitation spectrum at $\lambda_{\mathrm{em}}=480 \mathrm{~nm}$ [Fig. 3(A)] gives further evidence for ground-state dimer formation, as the changes observed are typical of pre-aggregation in the ground state, ${ }^{17,31}$ i.e. a small $(2-3 \mathrm{~nm})$ red shift and a lower peak to valley intensity ratio. These dimers are expected to originate from bound PMTP molecules, as the concentration of free PMTP in solution should be too low for dimer formation. The excitation of PMTP molecules bound through the nucleotides $\left(\lambda_{\mathrm{exc}}=260 \mathrm{~nm}\right)$ gives rise to an emission at $\sim 480 \mathrm{~nm}$, that is, lower than that obtained by direct excitation at $\lambda_{\text {exc }}=354 \mathrm{~nm}$. This can be easily understood as due to preferential excitation of intercalated PMTP through energy transfer from the polynucleotides at this shorter wavelength.

In single-strand polynucleotides, ${ }^{20}$ a maximum in the PMTP emission at $480 \mathrm{~nm}$ was observed at $[\mathrm{P}] /[\mathrm{D}]=1$, which decreased with a further increase in the amount of bases. This was attributed to external ionic binding that can induce PMTP sandwich-type complexes, ${ }^{20}$ which give rise to an excimer-like emission. As this marked effect is not seen in the double-strand polynucleotides, it can be concluded that, in this case, externally bound PMTP molecules do not cause the observed excimer-like emission. Thus, the observed dimers either come from the 

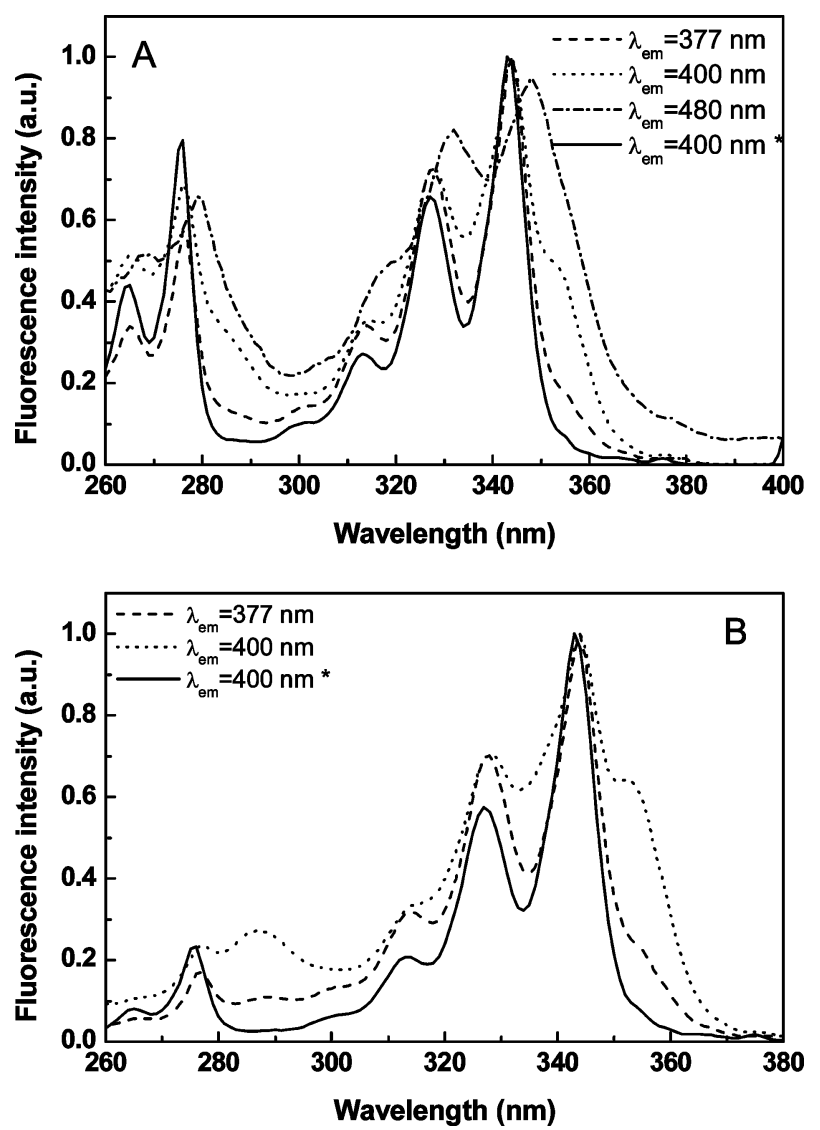

Fig. 3 Excitation spectra of PMTP $\left(1.5 \times 10^{-5} \mathrm{M}\right)$ in Poly[dA-dT] solution with $1 \times 10^{-3} \mathrm{M}$ phosphate buffer $(\mathrm{pH}$ 7.2) at different emission wavelengths: (A) without $\mathrm{NaCl}([\mathrm{P}] /[\mathrm{D}]=5)$; (B) with $0.1 \mathrm{M}$ $\mathrm{NaCl}([\mathrm{P}] /[\mathrm{D}]=30)$. Excitation spectra of free PMTP $(*)$ at $\lambda_{\mathrm{em}}=400$ $\mathrm{nm}$ are shown for comparison.

few unbound PMTP molecules or, more probably, from dimer formation at the grooves.

Fig. 3(B) shows the excitation spectra of $1.5 \times 10^{-5} \mathrm{M}$ PMTP in solution with Poly[dA-dT] in the presence of $0.1 \mathrm{M} \mathrm{NaCl}$. Under these conditions, we observe the same spectral behaviour, but only at a higher $[\mathrm{P}] /[\mathrm{D}]$ value $([\mathrm{P}] /[\mathrm{D}]=30)$. The presence of $\mathrm{NaCl}$ has a screening effect on the electrostatic binding of cationic PMTP with anionic phosphate groups, thus almost excluding external binding. This raises the $[\mathrm{P}] /[\mathrm{D}]$ ratio necessary to approach complete binding, either by intercalation and/or by groove binding. Furthermore, an increase in the ionic strength lowers the phosphate repulsion and thus induces a more compact polynucleotide structure. This fact probably increases the minimum number of bases required between consecutive intercalated PMTP molecules, due to size-exclusion effects.

The binding parameters were derived from the McGheevon Hippel treatment of the Scatchard isotherms, obtained from both absorption and fluorescence titrations. ${ }^{33}$ This was performed with and without the addition of $\mathrm{NaCl}(0.2 \mathrm{M})$. In the absence of $\mathrm{NaCl}$, the number of base pairs covered by one ligand $(n)$ indicated by both forms of analysis are similar (2.1 and 2.7 from fluorescence and absorption, respectively). The $K$ values were found to be $15 \times 10^{5}$ and $36 \times 10^{5} \mathrm{M}^{-1}$ from fluorescence and absorption, respectively, and together demonstrate the high affinity of the probe for the polynucleotide. However, this is reduced at high ionic strength $(0.2 \mathrm{M} \mathrm{NaCl})$ and the respective $K$ values obtained are $3.5 \times 10^{5}$ and $5.5 \times 10^{5} \mathrm{M}^{-1}$. The $n$ values from both the fluorescence and absorption titrations are also similar (6.0 and 8.6, respectively).

At high ionic strength, a significant diminution of excimerlike emission is observed in the fluorescence spectrum. As the $[\mathrm{P}] /[\mathrm{D}]$ ratio is six times higher in the presence of $\mathrm{NaCl}$, the local concentration of PMTP (the ratio between the number of PMTP molecules and the number of polynucleotide grooves in which dimerisation occurs) lowers.

In order to avoid the formation of aggregates between free PMTP molecules in the ground and excited states (dimers and excimers, respectively), a low concentration of PMTP $\left(2 \times 10^{-6}\right.$ $\mathrm{M})$ was used. For complete binding, in spectral terms, it was necessary to use a much higher $[\mathrm{P}] /[\mathrm{D}]$. This leads us to expect that there is no dimerisation between bound PMTP molecules, as their average separation should be larger.

Fig. 4 shows the emission spectra of a $2 \times 10^{-6} \mathrm{M}$ PMTPPoly[dA-dT] solution $([\mathrm{P}] /[\mathrm{D}]=110)$ recorded at several excitation wavelengths. Changing the ionic strength did not produce appreciable variations in the spectra. The observed spectral features are typical of the PMTP-polynucleotide bound species (and rather different from the free PMTP spectrum at $\lambda_{\mathrm{exc}}=354 \mathrm{~nm}$ ). However, small differences in the emission close to $400 \mathrm{~nm}$ can be seen. Also, small changes in the excitation spectra were observed for different emission wavelengths, indicating that several excited species are present. Emission in the excimer region is negligible for all the excitation wavelengths used, showing that the previously observed band around $480 \mathrm{~nm}$ at higher PMTP concentration (Fig. 2) mainly originates from pyrene-pyrene interactions, either in the ground or excited state. Furthermore, a clear distinction is observed between the excitation spectra of dilute and concentrated PMTP solutions (Fig. 3 and 4). In the dilute system, we obtain an excitation spectrum equivalent to the absorption spectrum, which is dominated by the complex of PMTP with Poly[dA-dT]. However, in the concentrated system, the absorption and excitation spectra do not coincide. This confirms the presence of PMTP dimers that have a negligible contribution to the absorption spectrum, but influence the excitation spectrum through its emission. The excitation spectrum in the dilute system allows us to confirm the polynucleotide sensitisation process through a broadening of the spectrum in the $250-290 \mathrm{~nm}$ region.

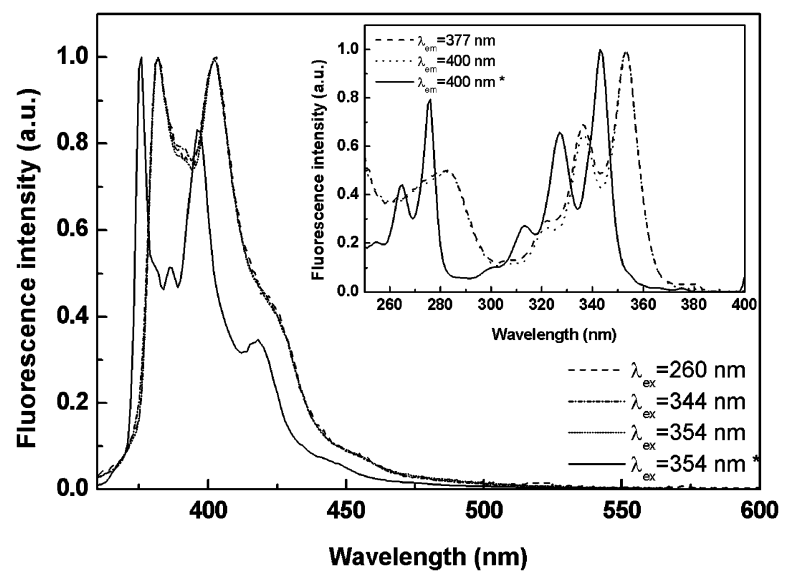

Fig. 4 Emission spectra of PMTP $\left(2 \times 10^{-6} \mathrm{M}\right)$ in Poly[dA-dT] solution $([\mathrm{P}] /[\mathrm{D}]=110)$ with $1 \times 10^{-3} \mathrm{M}$ phosphate buffer $(\mathrm{pH} 7.2)$, at different excitation wavelengths. The emission spectrum of free PMTP $\left.{ }^{*}\right)$ at $\lambda_{\mathrm{exc}}=354 \mathrm{~nm}$ is shown for comparison. The inset shows excitation spectra at different emission wavelengths. The excitation spectrum of free PMTP $(*)$ at $\lambda_{\mathrm{em}}=400 \mathrm{~nm}$ is shown for comparison.

Fluorescence quenching experiments using KI were performed in order to distinguish the binding modes and their relative accessibilities. The addition of $\mathrm{I}^{-}$to $2 \times 10^{-6} \mathrm{M}$ PMTP in Poly[dA-dT] (without $\mathrm{NaCl},[\mathrm{P}] /[\mathrm{D}]=110$ ) was analysed using a modified Stern-Volmer plot for the quenching of heterogeneous emitting sites: ${ }^{34,35}$

$$
\frac{I_{0}}{\Delta I}=\frac{1}{f_{\mathrm{a}}}+\frac{1}{f_{\mathrm{a}} K_{\mathrm{SV}}[\mathrm{Q}]}
$$


Table 2 Quenching by KI and fluorescence lifetime results for PMTP-Poly[dA-dT] in $1 \times 10^{-3} \mathrm{M}$ phosphate buffer (pH 7.2)

\begin{tabular}{|c|c|c|c|c|c|c|c|}
\hline$[\mathrm{PMTP}] / \mathrm{M}$ & {$[\mathrm{P}] /[\mathrm{D}]$} & $\lambda_{\mathrm{exc}} / \mathrm{nm}$ & $K_{\mathrm{sv}} / \mathrm{M}^{-1}$ & $f_{\mathrm{a}}$ & Fluorescence lifetime/ns & Pre-exponential values & $\chi^{2}$ \\
\hline $2 \times 10^{-6}(+\mathrm{KI})$ & 110 & $\begin{array}{l}344 \\
354\end{array}$ & $\begin{array}{l}74.5 \\
74.5\end{array}$ & $\begin{array}{l}0.24 \\
0.24\end{array}$ & & & \\
\hline $2 \times 10^{-6}$ & 110 & 354 & & & $\begin{array}{l}13.8 \\
46.4 \\
87.5\end{array}$ & $\begin{array}{l}0.12 \\
0.76 \\
0.12\end{array}$ & 1.1 \\
\hline $1.5 \times 10^{-5}(+\mathrm{KI})$ & 5 & $\begin{array}{l}344 \\
354\end{array}$ & $\begin{array}{l}570 \\
124\end{array}$ & $\begin{array}{l}0.88 \\
0.53\end{array}$ & & & \\
\hline $1.5 \times 10^{-5}$ & 5 & 354 & & & $\begin{array}{c}13.8 \\
46.4 \\
87.5 \\
123\end{array}$ & $\begin{array}{l}0.11^{a} \\
0.63^{a} \\
0.26^{a} \\
0.103\end{array}$ & 1.1 \\
\hline
\end{tabular}

where $K_{\mathbf{S V}}$ is the Stern-Volmer constant, $f_{\mathrm{a}}$ is the accessibility of the quencher molecule, $\mathrm{Q}$, and $[\mathrm{Q}]$ is the quencher concentration. For the excitation wavelengths of 344 and $354 \mathrm{~nm}$, the calculated accessibility and $K_{\mathrm{Sv}}$ are the same (Table 2). This result shows that $76 \%$ of emitting sites are located inside the double helix and, thus, are not accessible to the quencher, implying that intercalative binding is the preferred form of PMTP-Poly[dA-dT] association.

The fluorescence decay of PMTP + Poly[dA-dT] was found to be multiexponential and the decay analysis is presented in Table 2. These data provide evidence for the presence of several excited-state species in the polynucleotide solution. The emission spectrum of this complex (see Fig. 4) indicates that all PMTP is bound to the polynucleotide, which is confirmed by the absence of the lifetime component at about 130 ns relating to free PMTP in solution. The three decay components can be ascribed to different binding modes of PMTP with Poly[dA-dT].

It is known that the pyrene-AMP complex has a relatively long lifetime (138 ns), whereas the pyrene-TMP complex has a short lifetime (5.8 ns). ${ }^{6}$ As the interaction between pyrene and the nucleotides is mainly determined by $\pi-\pi$ interactions, we expect to find the same general trends for PMTP. However, it should be noted that the attractive electrostatic interaction between PMTP and the nucleotide phosphate group allows a more favourable approach between the two molecules. Nevertheless, some preliminary results ${ }^{33}$ indicate that PMTPTMP has a fluorescence lifetime of $7.1 \mathrm{~ns}$, which is similar to the published value for pyrene-TMP. ${ }^{6}$ Also, the decay of PMTP in a single-strand Poly[dA] complex was found to have a main component of 119.1 ns. $^{20}$

When PMTP is intercalated in Poly[dA-dT], it contacts directly with adenine and thymine, giving rise to an intermediate PMTP decay time of $\sim 45$ ns. This component represents $76 \%$ of the decay, in very good agreement with the accessibility predicted by the quenching results. The 13.8 and 87.5 ns components arise from surface binding. As the pyrene moiety of PMTP has a hydrophobic character, we can expect that the surface-bound PMTP orientates its pyrene group inside the double strand, approaching the bases. Therefore, the shorter component can be ascribed to weak binding sites closer to thymine nucleotides and the longer one to binding sites closer to adenine nucleotides. It should be noted that the weight of these two decay components is similar, in accordance with the polynucleotide composition.

The higher accessibility and $K_{\mathrm{Sv}}$ value when $\lambda_{\mathrm{exc}}=344 \mathrm{~nm}$ and KI is added to Poly[dA-dT]-PMTP $\left(1.5 \times 10^{-5} \mathrm{M}\right)([\mathrm{P}] /$ $[\mathrm{D}]=5$ ) in the absence of $\mathrm{NaCl}$ (Table 2) is another indication that free or weakly bound PMTP is important in this more concentrated system. The fluorescence decay of this system (at $\lambda_{\text {exc }}=354 \mathrm{~nm}$ ) was analysed by fixing the decay times that we obtained previously for the bound PMTP states. From this analysis, we identified an extra component with a lifetime of
123 ns $(10.3 \%)$, which we attribute to free PMTP. This would predict an accessibility at $\lambda_{\text {exc }}=354 \mathrm{~nm}$ of, at most, $43 \%$ [sum of the weights of the components due to non-intercalated species: $0.103+(1-0.103) \times(1-0.63)]$. The difference from the quenching results could be because of the influence on the decay of the component near 53 ns, which is observed for $1.5 \times 10^{-5}$ M PMTP in aqueous solution (without polynucleotide). This could increase the weight of the $46 \mathrm{~ns}$ component. However, it should be pointed out that a detailed kinetic analysis of this system is complex because of the presence of multiple types of bound species and the superposition of unbound PMTP excited-state kinetics. The quenching by iodide of $1.5 \times 10^{-5} \mathrm{M}$ PMTP-Poly[dA-dT] $-0.1 \mathrm{M} \mathrm{NaCl}$ solution $([\mathrm{P}] /[\mathrm{D}]=30)$ gives rise to quenching constants similar to those obtained in the absence of $\mathrm{NaCl}$. In the presence of $0.1 \mathrm{M} \mathrm{NaCl}$, electrostatic binding is not significant; thus, considering the same level of PMTP binding (higher $[\mathrm{P}] /[\mathrm{D}]$ value), an enhanced fraction of intercalation takes place, with a corresponding decrease in accessibility to quencher.

The steady-state anisotropy of $2 \times 10^{-6}$ M PMTP-Poly[dA-dT] (in the absence of $\mathrm{NaCl}$ and at $[\mathrm{P}] /[\mathrm{D}]=110$ ) using excitation wavelengths of 344 and $354 \mathrm{~nm}$ returns a value of $r=0.1$, independent of the emission wavelength in the region $390-460$. As the pyrene moiety is planar, it is expected that the intrinsic fluorescence anisotropy should be near 0.4. The rotation of the pyrenyl moiety of intercalated PMTP is almost completely hindered. Therefore, if only the intercalated PMTP molecules (which constitute about $76 \%$ of the total number of PMTP molecules) contribute to the observed anisotropy, this value should be at least 0.28 . The additional depolarisation must come either from rotations of the polynucleotide helix or from energy transfer to other nearby PMTP molecules. The first mechanism should not contribute significantly as the lifetime of intercalated PMTP molecules is $\sim 45 \mathrm{~ns}$ and the DNA rotation time is near $1 \mathrm{~s}^{36}$

At high probe concentrations, the anisotropy changes from 0.1 when the excitation wavelength is $354 \mathrm{~nm}$ to $\sim 0.03$ when the excitation wavelength is $344 \mathrm{~nm}$. This can be explained by the presence of free PMTP, which is preferentially excited at 344 $\mathrm{nm}$ and contributes with an anisotropy near zero (as bulk water provides a low viscosity environment). The anisotropy results at high PMTP and $\mathrm{NaCl}$ concentrations are in agreement with the iodide quenching results.

PMTP-Poly[dA] Poly[dT] interactions. Solutions of PMTP in Poly[dA] Poly[dT] at low ionic strength also exhibit a $9 \mathrm{~nm}$ red shift in their absorption spectra compared to PMTP in aqueous solution. There is also the hypochromism observed in the case of Poly[dA-dT], but at a lower $[\mathrm{P}] /[\mathrm{D}]$ value $([\mathrm{P}] /[\mathrm{D}]=$ $3)$. In the presence of $0.2 \mathrm{M} \mathrm{NaCl}$, these effects are strongly reduced (for example, at $[\mathrm{P}] /[\mathrm{D}]=14.6$, the hypochromism is $\sim 12 \%$ and only a small shoulder at $353 \mathrm{~nm}$ is observed). This 
indicates that the level of intercalation is low and explains the lower value of $[\mathrm{P}] /[\mathrm{D}]$ needed for PMTP binding. The unfavourable intercalation of PMTP in Poly[dA]·Poly[dT] could be a result of a more compact structure, as this polynucleotide adopts the A form instead of the usual B form. ${ }^{37}$

The fluorescence intensity of PMTP-Poly[dA]·Poly[dT] solution at low ionic strength is reduced to $20 \%$ of that of free $\operatorname{PMTP}\left(\lambda_{\mathrm{em}}=400 \mathrm{~nm}\right)$ at low $[\mathrm{P}] /[\mathrm{D}]$ values, but when $[\mathrm{P}] /[\mathrm{D}]$ is high, the fluorescence intensity rises again and a red shift of $4 \mathrm{~nm}$ is observed, which is typical for the binding of PMTP with A-A bases. ${ }^{20}$ However, in this double-strand polynucleotide, the excimer-like emission is not observed, probably as the higher rigidity of this polynucleotide prevents the formation of sandwich PMTP dimers. The fluorescence is dominated by the PMTP-adenine interaction, which exhibits significant red shifts in both the fluorescence and absorption maxima, but shows a low quenching efficiency. In contrast, with thymine sites, PMTP is efficiently quenched with very small spectroscopic variations. ${ }^{6}$ The peculiar variation in the emission intensity can be explained by processes that depend on local concentration. In the initial stage of binding, PMTP molecules are in excess and, thus, the polynucleotide surfaces are almost completely covered, which results in a high local concentration. As the polynucleotide concentration is increased, the bound PMTP molecules get further apart and, thus, the local concentration decreases. Processes depending on local concentration could be either the formation of a non-emitting dimer on the polynucleotide surface or an energy-transfer process from a PMTP molecule close to an adenine base (inefficient quenching) or to a PMTP molecule close to a thymine base (efficient quenching).

In the presence of $0.2 \mathrm{M} \mathrm{NaCl}$, there is only a small decrease in the fluorescence intensity $(25 \%)$ and no significant red shift was detected. This fact confirms that weak binding (external and/or groove) is the predominant mechanism for this polynucleotide and is strongly affected by the presence of $\mathrm{NaCl}$. Furthermore, it seems that thymine sites are more resistant to $\mathrm{NaCl}$, as there is no red shift in the emission.

The fluorescence decay of the complex in the absence of $\mathrm{NaCl}$ was found to be triexponential, with components of $18.5(16 \%)$, $40.6(72 \%)$ and $123 \mathrm{~ns}(12 \%)$. Although the lifetimes are similar to those observed in Poly[dA-dT], their correspondence with the type of binding is not so straightforward. The weak binding sites can now be AAA or TTT, which contribute with a long ( $119.1 \mathrm{~ns}$ for PMTP-Poly[A] complex) and a short lifetime ( $\sim 7$ ns for PMTP-thymidine complex), respectively. Note that the long lifetime can be influenced by the presence of any residual free PMTP molecules. The intermediate lifetime is similar to that attributed to AT intercalation in the heteropolymer. This lifetime mainly reflects PMTP in ATA-type sites in the grooves (minor or major). Shafirovich et al. ${ }^{38}$ found that thymine quenches pyrene derivatives through a proton-coupled oxidative photoinduced electron transfer. In intercalation sites, we expect a lower accessibility to protons. Thus, the few intercalated PMTP molecules would contribute to the intermediate lifetime.

PMTP-Poly[dG-dC] interactions. Fig. 5 shows absorption (inset), excitation and emission spectra of PMTP $\left(1.5 \times 10^{-5}\right.$ $\mathrm{M})$ in Poly[dG-dC] solution. The absorption also exhibits a red shift with increasing $[\mathrm{P}] /[\mathrm{D}]$, as verified for Poly[dA-dT] and Poly[dA]·Poly[dT]. Hypochromism was also detected, but required a higher $[\mathrm{P}] /[\mathrm{D}]$ value to obtain similar levels. The higher $[\mathrm{P}] /[\mathrm{D}]$ needed for complete binding shows that PMTP intercalates in this heteropolynucleotide, although not as efficiently as in Poly[dA-dT].

In this case, a clear isobestic point in the absorption spectra is not observed. This strongly suggests that another ground-state species is playing a role.

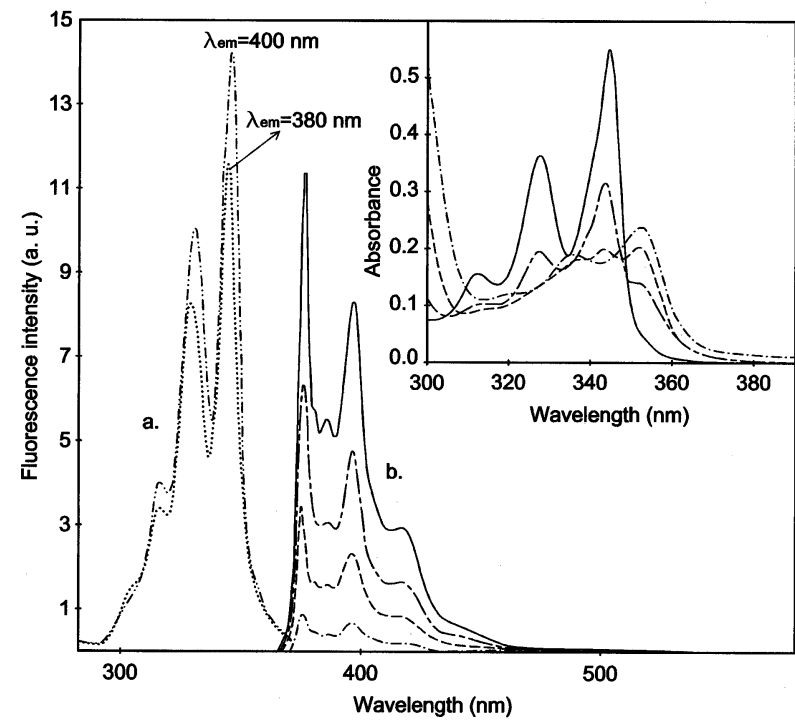

Fig. 5 (a) Excitation spectra for PMTP $\left(1.5 \times 10^{-5} \mathrm{M}\right)$ in Poly[dG-dC] solution with $1 \times 10^{-3} \mathrm{M}$ phosphate buffer $(\mathrm{pH} 7.2)$ at $[\mathrm{P}] /[\mathrm{D}]=73: \lambda_{\mathrm{em}}=$ $400(-\cdot-), 380 \mathrm{~nm}(\cdots)$. (b) Emission spectra $\left(\lambda_{\mathrm{exc}}=350 \mathrm{~nm}\right)$ for PMTP $\left(1.5 \times 10^{-5} \mathrm{M}\right)$ in Poly[dG-dC] solution with $1 \times 10^{-3} \mathrm{M}$ phosphate buffer (pH 7.2) at different $[\mathrm{P}] /[\mathrm{D}]$ ratios: $(-) 0$; (- - ) 14; (--) 25; $(--)$ 73. The inset shows absorption spectra for the same PMTPPoly[dG-dC] solutions.

The emission spectra [Fig. 5(b)] show a reduction of about $90 \%$ in the fluorescence intensity. However, no appreciable red shift or changes in band shape are observed, even at very high $[\mathrm{P}] /[\mathrm{D}]$. Also, no emission was observed at longer wavelengths. The excitation spectrum at $\lambda_{\mathrm{em}}=400 \mathrm{~nm}$ is similar to that at $\lambda_{\mathrm{em}}=380 \mathrm{~nm}$. Therefore, the formation of a complex between PMTP and Poly[dG-dC] causes no appreciable spectral changes to the emission, besides the reduction in intensity, thus giving support for the existence of a less emissive species. Lianos and Georghiou $^{6}$ reported similar emission behaviour for the pyrene-CMP complex, its spectra resemble those of free pyrene, and also very slight changes in the absorption spectrum ( $\sim 0.7 \mathrm{~nm}$ red shift). The fluorescence quenching of pyrenyl rings by guanine has been studied ${ }^{16,38-41}$ and this phenomenon attributed to a reductive photoinduced electron-transfer mechanism. ${ }^{15,38-41}$ Because of the close proximity between guanine and PMTP in Poly[dG-dC], this photoinduced electron transfer can occur so fast that no emission is detectable from those PMTP molecules that are quenched by guanine nucleotides. In contrast to the situation with cytosine, the pyrene complex with guanine exhibits marked changes in its absorption spectra. ${ }^{6}$ Thus, the spectral characteristics of PMTP in Poly[dG-dC] solution are determined by guanine in absorption and by cytosine in emission.

The intercalative complex between $\mathrm{CG}$ can be observed in absorption, but not in emission, where it contributes only as an emission trap. The emitting species at the 'end' of the binding experiment are determined by those bound PMTP molecules that are sufficiently far from guanine (external and/or groove binding). As in the previous cases, there is an absence of excimer-like emission from external binding. This fact is due to the rigid 3D structure of the double strand, as compared to the single strand. ${ }^{20}$

As time-resolved fluorescence measurements are very sensitive to excited-state kinetics, decay curves can elucidate more information about excited species in Poly[dG-dC] solution. The fluorescence decay in the absence of $\mathrm{NaCl}$ was found to be biexponential, with lifetimes of $5.1(5 \%)$ and $122 \mathrm{~ns}(95 \%)$. The short component in the former probably arises from bound PMTP molecules in which the pyrene moiety is near to cytosine (the pyrene-CMP complex has a short lifetime of $9.5 \mathrm{~ns}$ ). ${ }^{6}$ These PMTP molecules could be bound either externally or in 
Table 3 Relative minimum energies $\left(\mathrm{kcal} \mathrm{mol}^{-1}\right)$ obtained from molecular modelling after geometry optimisation of a PMTP molecule situated in different binding sites on a thirty base-pair polynucleotide structure. The energies are also given for PMTP and the polynucleotide when separated (not interacting). The force field employed was AMBER2

Binding position

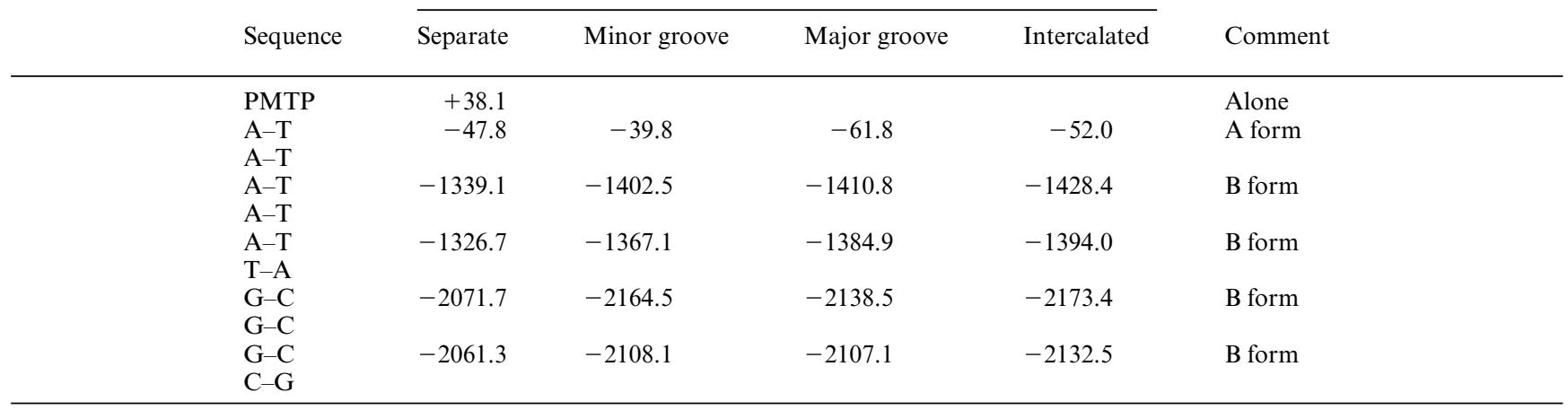

grooves. The longer component is similar to that of free PMTP, thus, the decay is apparently dominated by free PMTP, showing that most of the bound molecules are near guanine and are, therefore, efficiently quenched.

PMTP-Poly[dG]·Poly[dC] interactions. The absorption spectrum of PMTP in Poly[dG]·Poly[dC] solution in the absence of $\mathrm{NaCl}$ shows only a shoulder at $353 \mathrm{~nm}$ and has no distinct isobestic point, while the hypochromism is about $25 \%$ at $[\mathrm{P}] /[\mathrm{D}]=12$. In the presence of $0.2 \mathrm{M}$ sodium chloride, at the same PMTP concentration and $[\mathrm{P}] /[\mathrm{D}]$ ratio, there is only a broadening in the longer wavelength region of the absorption and the hypochromism is negligible $(\sim 6 \%)$. This fact indicates either a lower degree of intercalation than in the corresponding heteropolymer or the presence of intercalation sites with negligible spectral variations in absorption, such as $\mathrm{C}-\mathrm{C}$ intercalation sites.

In the absence of $\mathrm{NaCl}$, similar fluorescence spectral behaviour to that already seen with the heteropolymer Poly[dG$\mathrm{dC}]$ at $[\mathrm{P}] /[\mathrm{D}]=73$ is observed. However, the homopolymer requires a lower $[\mathrm{P}] /[\mathrm{D}]$ value $([\mathrm{P}] /[\mathrm{D}]=12)$ to reduce the fluorescence intensity to $20 \%$ of the intensity of free PMTP at $400 \mathrm{~nm}$. This can be explained by the greater affinity of PMTP for GG than CC sites, as already seen with single-strand polynucleotides. ${ }^{20}$ As previously stated, GG intercalation sites are not emissive. $\mathrm{CC}$ intercalation sites are also expected to show a low emission, as there are two $G$ residues almost at contact distance, making guanine quenching very efficient. Note that, as in the thymine case, we expect the proton-assisted oxidative quenching of PMTP by cytosine to be less efficient in intercalation sites than it is in water. Therefore, the observed efficient quenching of PMTP in the homopolymer is, in the main, determined by weakly bound PMTP molecules. This effect can originate either from an enhanced quenching or from an enhanced binding to GGG external sites, as compared to GCG ones.

In the presence of $0.2 \mathrm{M}$ sodium chloride, we observed a reduction in the fluorescence intensity to $80 \%$ that of free PMTP, with the vibrational structure of emission maintained However, a significant excimer-like band appears in the fluorescence spectrum when $353 \mathrm{~nm}$ excitation is used. The fact that this band is negligible upon excitation at $344 \mathrm{~nm}$ excludes the possibility of a dynamic excimer or an exciplex formed through the reaction of an excited monomer with another molecule in the ground state. This behaviour is typical of the presence of a ground-state pyrene dimer, ${ }^{28,31}$ which is preferentially excited in the longer wavelength region (where monomer absorption is very low) and can revert rapidly to an excimer-like species in solution. This static excimer is formed by internal conversion of the excited pre-associated dimer. ${ }^{31}$ The addition of KI to $1.5 \times 10^{-5}$ M PMTP-Poly[dG] Poly[dC] $-0.1 \mathrm{M} \mathrm{NaCl}$ solution $([\mathrm{P}] /[\mathrm{D}]=30)$ produces a quenching constant of $K_{\mathrm{Sv}}=430 \mathrm{M}^{-1}$ at both $\lambda_{\mathrm{exc}}=344$ and $353 \mathrm{~nm}$. The calculated accessibilities are $100 \%$ at $344 \mathrm{~nm}$ and $83 \%$ at $353 \mathrm{~nm}$, confirming a low level of intercalation, although only the $\mathrm{C}-\mathrm{C}$ sites are detectable with this assay.

For the homopolymer, in absence of $\mathrm{NaCl}$, the fluorescence decay required the sum of three exponential components to fit to it, with lifetimes $5(6 \%), 53(14 \%)$ and $138 \mathrm{~ns}(80 \%)$. The first component was observed in Poly[dG-dC] and is ascribed to PMTP-cytosine interactions. The second component relates to the emission of sandwich-like PMTP dimers that can revert to excimers upon excitation. In aqueous media, these kinetics correspond to the 63 and 2.2 ns decay times, the latter probably included in the observed short lifetime (5 ns) component. PMTP in CC intercalation sites probably contributes to the intermediate component through nearby guanine quenching. The long component is typical of free PMTP in aqueous solution. As in Poly[dG-dC], the decay is dominated by free PMTP, but this does not mean that PMTP molecules are not binding to polynucleotide sites. The problem is that all bound PMTP molecules that are near guanine are very efficiently quenched and, thus, are a dark species.

As the excimer-like emission is significantly enhanced in the presence of $\mathrm{NaCl}$, we can conclude that it cannot arise from externally bound PMTP. Therefore, the dimerisation process occurs at the grooves of the polynucleotide.

Molecular modelling. Although the evidence of intercalation and groove binding from the spectroscopic studies appears very strong, further conformation of PMTP polynucleotide interactions was obtained through the use of some basic molecular modelling. Structures containing a thirty base-pair doublestrand sequence (for example, either alternating bases on one strand or using the same base on one strand) were geometrically optimised before a geometrically optimised PMTP structure was placed in the desired binding location. A further geometry optimisation was then run and the energy of the combined structures calculated. To check that the value obtained did not reflect a local energy minimum, an annealing procedure was performed and the geometry optimised again. The outcome of these calculations is presented in Table 3 .

These data show that, overall, the energies of the interacting species are less than those of the non-interacting ones. The exception is when using the $\mathrm{A}$ and $\mathrm{T}$ bases fixed in an $\mathrm{A}$ form of compact helix; here, the modelling appears to be ambiguous. However, if the helix is in the looser B form, then interactions are favoured in all cases. The trend also appears to show that the most energetically favoured form of interaction between the polynucleotides and PMTP is that of intercalation. These results are in keeping with those that we observed experimentally. 


\section{Summary and conclusions}

The positively charged pyrene derivative PMTP has the advantage of being water soluble, while retaining the environmental sensitivity of pyrene. The pyrene moiety gives it the ability to intercalate, while the positive charge, in conjunction with the anchor group (the butyl chains), contribute to the weak binding interaction (external and groove). It should be noted, however, that the anchor group does not allow complete intercalation, which permits PMTP to discriminate between the double helix and a single strand. ${ }^{20}$

PMTP, upon interaction with polynucleotides, exhibits changes in its absorption spectrum (hypochromicity and red shift) and a reduction in its fluorescence intensity. Thymine and cytosine bases quench PMTP by a proton-assisted oxidative electron transfer. Guanine base quenches PMTP by a reductive electron transfer, while adenine does not quench PMTP. These known processes allow the location and form of binding of PMTP to be distinguished through time-resolved fluorescence measurements. Namely, $\mathrm{T}$ and $\mathrm{C}$ surface sites have good proton accessibility, while intercalation sites are more protected. In heteropolynucleotides, the sites are more homogeneous (ATATAT or GCGCGC) than in the corresponding homopolynucleotides (AAATTT or GGGCCC). Thus, this probe has the ability to distinguish homosequences from heterosequences. Ground-state interactions give also evidence of the preferences of PMTP in terms of sequence, structure and type of binding.

Also of importance are bimolecular PMTP processes on the polynucleotide surface. We observed that PMTP dimerises, giving rise to an excimer-like emission (groove binding), and is also involved in direct energy transfer (DET) between several bound PMTP molecules. The latter was observed as a hopping of excitation energy from inefficient quenching sites (adenine) to efficient ones (thymine). The dimerisation process enhances charge transport in polynucleotides mediated by PMTP. The direct energy transfer moves the excitation energy across the polynucleotide. Both processes are important in the development of DNA-based biosensors, making use of its charge and excitation energy hopping abilities.

To summarise, this work confirms that the positively charged pyrene derivative PMTP exhibits the ability to bind to nucleic acids, both by intercalation between the bases and by groove binding. These are distinguishable spectroscopically and, thus, this probe complements other studies making use of pyrene derivatives.

\section{Abbreviations \\ A, adenine; AMP, adenosine monophosphate; C, cytosine; CMP, cytidine monophosphate; EDTA, ethylenediaminetetra- acetic acid; G, guanine; GMP, guanidine monophosphate; PMTP, pyrenylmethyl tri-n-butylphosphonium bromide; Poly[dA-dT], double-strand polynucleotide Poly[dA-dT] Poly[dA-dT]; Poly[dG-dC], double-strand polynucleotide Poly[dG-dC]·Poly[dG-dC]; T, thymine; TMP, thymidine monophosphate.}

\section{References}

$1 \mathrm{H}$. Weil-Malherbe, The solubilization of polycyclic aromatic hydrocarbons by purines, Biochem. J., 1946, 40, 351-363.

2 E. Boyland and B. Green, On the reported sedimentation of polycyclic hydrocarbons from aqueous solutions of DNA, J. Mol. Biol., 1964, 9, 589-597.

3 J. K. Ball, J. A. McCarter and M. F. Smith, The interaction in vitro of polycyclic aromatic hydrocarbons with deoxyribonucleic acids, Biochim. Biophys. Acta, 1965, 103, 275-285.

4 A. R. Peacocke, in Heterocyclic Compounds: Acridines, ed. R. M. Acheson, Interscience, New York, 1973, p. 723.

5 N. E. Geacintov, T. Prusik and J. M. Khosrofian, Properties of benzopyrene-DNA complexes investigated by fluorescence and triplet flash photolysis techniques, J. Am. Chem. Soc., 1976, 98, 6444-6452.

6 P. Lianos and S. Georghiou, Complex formation between pyrene and the nucleotides GMP, CMP, TMP and AMP, Photochem. Photobiol., 1979, 29, 13-21.

7 N. E. Geacintov, D. Zinger, V. Ibanez, R. Santella, D. Grunberger and R. G. Harvey, Properties of covalent benzo[a]pyrene diol epoxide-DNA adducts investigated by fluorescence techniques, Carcinogenesis, 1987, 8, 925-935.

8 N. E. Geacintov, M. Cosman, B. Mao, V. Ibanez and R. G. Harvey, Spectroscopic characteristics and site I/II classification of cis and trans benzo[a]pyrene diol epoxide enantiomer-guanosine adducts in oligonucleotides and polynucleotides, Carcinogenesis, 1991, 12, 2099-2108.

9 C. V. Kumar, R. S. Turner and E. H. Asuncion, Groove binding of a styrylcyanine dye to the DNA double helix: the salt effect, J. Photochem. Photobiol., A, 1993, 74, 231-238.

10 N. E. Geacintov, R. Zhao, V. A. Kuzmin, S. K. Kim and L. J. Pecora, Mechanism of quenching of the fluorescence of a benzo[a]pyrene tetraol metabolite model compound by $2^{\prime}$-deoxynucleosides, Photochem. Photobiol., 1993, 58, 185-194.

11 Y. Cao and H. Xi-men, Studies of interaction between Safranine T and double strand helix DNA by spectral methods, Spectrochim. Acta, Part A, 1998, 54, 883-892.

12 H. C. Becker and B. Nordén, DNA binding mode and sequence specificity of piperrazinecarbonyloxyalkyl derivatives of anthracene and pyrene, J. Am. Chem. Soc., 1999, 121, 11947-11952.

13 H. C. Becker and B. Nordén, DNA binding thermodynamics and sequence specificity of chyral piperrazinecarbonyloxyalkyl derivatives of anthracene and pyrene, J. Am. Chem. Soc., 2000, 122, $8344-8349$.

14 C. V. Kumar, E. H. A. Punzalan and W. B. Tan, Adenine-thiamine base pair recognition by an anthryl probe from the DNA minor groove, Tetrahedron, 2000, 56, 7027-7040.

15 N. E. Geacintov, M. Shahbaz, V. Ibanez, K. Moussaoui and R. G. Harvey, Base-sequence dependence of noncovalent complex formation and reactivity of benzo[a]pyrene diol epoxide with polynucleotides, Biochemistry, 1988, 27, 8380-8387.

16 M. E. C. D. Real Oliveira, J. A. Ferreira, S. M. Nascimento, H. D. Burrows and M. G. Miguel, Oligomerization of cationic pyrene derivatives in the presence of sodium dodecyl sulfate: a route to nanoaggregate formation, J. Chem. Soc., Faraday Trans., 1995, 91, 3913-3917.

17 E. Zahavy and M. A. Fox, Photophysical quenching mediated by guanine groups in pyrenyl-N-alkylbutanoamide end-labeled oligonucleotides, J. Phys. Chem. B, 1999, 103, 9321-9327.

18 G. B. Behera, B. K. Mishra, P. K. Behera and M. Panda, Fluorescent probes for structural and distance effects studies in micelles, reverse micelles and microemulsions, Adv. Colloid Interface Sci., 1999, 82, $1-42$.

19 M. E. C. D. Real Oliveira, H. D. Burrows, M. G. Miguel and G. R. Jones, Application of fluorescence probe pyrenylmethyltri-n-butyl phosphonium bromide (PMTP) in studies of micelles polyelectrolites and polynucleotides, J. Fluoresc., 1997, 7, 191S$193 \mathrm{~S}$.

20 G. R. Jones, M. E. C. D. R. Oliveira and R. B. Cundall, The interactions of pyrenyl tributylphosphonium bromide with single strand polynucleotides, Photochem. Photobiol., 1990, 52, 451-460.

21 M. L. D'Amico, V. Paiotta, F. Secco and M. Venturini, A kinetic study of the intercalation of ethidium bromide into Poly(A).Poly(U), J. Phys. Chem. B, 2002, 106, 12635-12641.

22 L. S. Ling, Z. K. He and Y. E. Zeng, Spectral studies on the interaction of DNA and Ru(bipy) ${ }_{2}(\mathrm{dppx})^{2+}$, Spectrochim. Acta, Part A, 1999, 55, 1297-1302.

23 E. Renault, M. P. Fontaine-Aupart, F. Tfibel, M. Gardes-Albert and E. Bisagni, Spectroscopic study of the interaction of pazelliptine with nucleic acids, J. Photochem. Photobiol., B, 1997, 40, 218-227.

24 M. E. C. D. R. Oliveira, L. C. Pereira, E. W. Thomas, R. H. Bisby and R. B. Cundall, Photochemistry of pyrenylmethyltriphenylphosphonium salts and related compounds, J. Photochem., 1985, 31, 373-379.

25 J. D. McGhee and P. H. von Hippel, Theoretical aspects of DNA-protein interactions: Co-operative and non-co-operative binding of ligands to a one dimensional homogeneous lattice, J. Mol. Biol., 1974, 86, 469-489.

26 D. C. Dong and M. A. Winnik, The Py scale of solvent polarities, Can. J. Chem., 1984, 62, 2560-2565.

27 E. M. S. Castanheira, J. M. G. Martinho, D. Duracher, M. T. Charreyre, A. Elaïssari and C. Pichot, Study of cationic $\mathrm{N}$-isopropylacrylamide-styrene copolymer latex particles using fluorescent probes, Langmuir, 1999, 15, 6712-6718. 
28 J. M. G. Martinho, E. M. S. Castanheira, A. T. Reis e Sousa, S. Saghbini, J. C. André and M. A. Winnik, Polystyrene cyclization under high hydrostatic pressure, Macromolecules, 1995, 28, 1167-1171.

29 S. Hashimoto, Mechanism of fluorescence quenching of pyrene with purines in polar media. Formation of the pyrene triplet state via exciplex formation, J. Phys. Chem., 1993, 97, 3662-3667.

$30 \mathrm{H}$. P. Nelson and H. DeVoe, Physical binding of pyrene and phenanthrene to native and denatured DNA: measurements by spectral and coupled-column liquid chromatography methods, Biopolymers, 1984, 23, 897-911.

31 F. M. Winnik, Photophysics of preassociated pyrenes in aqueous polymer solutions and in other organized media, Chem. Rev., 1993, 93, 587-614.

32 C. R. Cantor and P. R. Schimmel, Biophysical Chemistry, Part II Techniques for the Study of Biological Structure and Function, Freeman and Co., New York, 1980, p. 349.

33 M. E. C. D. R. Oliveira, A Photophysical and Photochemical Study of Some Pyrenyl Phosphonium Salts, PhD Thesis, University of Salford, UK, 1986.

34 S. S. Leher, Solute perturbation of protein fluorescence. The quenching of the tryptophyl fluorescence model compounds and of lysosyme by iodide ion, Biochemistry, 1971, 10, 3254-3263.

35 B. Valeur, Molecular Fluorescence - Principles and Applications, Wiley-VCH, Weinheim, 2002, p. 72.
36 Y. Harada, O. Ohara, A. Takatsuki, H. Itoh, N. Shimamoto and K. Kinosita Jr., Direct observation of DNA rotation during transcription by Escherichia coli RNA polymerase, Nature, 2001, 409, 113-115.

37 G. Ge and S. Georghiou, Room temperature fluorescence properties of the polynucleotide Poly[dA].Poly[dT], Photochem. Photobiol., 1991, 54, 477-480.

38 V. Y. Shafirovich, S. H. Courtney, N. Ya and N. E. Geacintov, Proton-coupled photoinduced electron transfer, deuterium isotope effects, and fluorescence quenching in noncovalent benzo[a]pyrenetetraol-nucleoside complexes in aqueous solutions, J. Am. Chem. Soc., 1995, 117, 4920-4929.

39 C. Kerr, D. C. Mitchell, Y. Ying, B. E. Eaton and T. L. Netzel, Synthesis and photophysics of a 1-pyrenylmethyl-substituted 2'deoxyuridine-5-carboxamide nucleoside: electron-transfer product lifetimes and energies, J. Phys. Chem. B, 2000, 104, 2166-2175.

40 V. Y. Shafirovich, P. P. Levin, V. A. Kuzmin, T. E. Thorgeirsson, D. S. Kliger and N. E. Geacintov, Photoinduced electron transfer and enhanced triplet yields in benzo[a]pyrene derivative-nucleic acid complexes and covalent adducts, J. Am. Chem. Soc., 1994, 116, 63-72.

41 N. E. Geacintov, V. Y. Shafirovich, B. Li, B. Mao and N. Ya, Photoinduced electron transfer, fluorescence, and intrastrand migration of reactive intermediates in pyrenyl sensitizer - modified DNA duplexes, The Spectrum, 1997, 10, 1-9. 